\title{
Targeting the Epigenetic Factors of Neuroinflammation
}

\author{
Anusha Patil
}

Written under the direction of Dr. David P. Crockett

\begin{abstract}
Currently, there are no effective treatments for traumatic brain injury (TBI). This is because the mechanisms behind post-injury neuroinflammation are not well understood. This project studies a novel signaling pathway responsible for the activation of microglia post-TBI. Its goal is to identify epigenetic factors of neuroinflammation that may be targeted with future therapies.
\end{abstract}

We are examining the possibility that class Ila Histone Deacetylases (HDACs), particularly HDAC7, are responsible for initiating the inflammatory response after TBI. In addition, we plan to explore what its upstream regulation factors may be. One possible upstream regulation factor explored is regulating Kinase 2, also known as Parlb (Par1b/MARK2), since prior research indicates that a deficiency in Par1b/MARK2 increased the inflammatory response of microglia in a mouse model of TBI. In our experiments, we examined brains from sham mice (i.e., without head injury) and mice subjected to closed head injury (CHI). Our experiments made use of wild-type mice and mice deficient in Par1b/MARK2. Qualitative analyses were conducted using fluorescent microscopy imaging of immunohistochemistry. Using cell-specific markers of inflammation, we found an increase in astrocytic marker GFAP (glial fibrillary acidic protein) and microglial protein IBA1 (ionized calcium binding protein) expression in the cortex of the mice after $\mathrm{CHI}$. These increases were dramatic ipsilaterally (same side) to the injury, but only moderate contralaterally (opposite side). In the control brains (sham operates), little to no increase in these markers were detected.

In our experiments, we observed increased expression of HDAC7 in post-TBI microglia as well as in reactive GFAP-expressing astrocytes. Others have observed that Par1b/MARK2 may negatively regulate HDAC7 activity and there is evidence that HDAC7 is an inhibitor of antiinflammatory genes. 


\section{Introduction}

Traumatic brain injury (TBI) has been dubbed the "silent epidemic." It is estimated that 69 million people worldwide suffer from death and disability caused by TBI, which is more than any other traumatic insult (Dewan et al 2019). In the U.S. alone, approximately 1.7 million people sustain a TBI each year, which leads to at least 52,000 deaths annually. The financial burden on society in both direct and indirect costs adds up to an astounding $\$ 60$ billion, not to mention the cost of the individual's life or independence. Unfortunately, there is currently no effective treatment for TBI. One major reason for this is the body's inflammatory response that follows damage to the central nervous system (e.g., Loane and Byrnes 2010; Faden and Loane, 2015). Neuroinflammation is a double-edged sword because it has both beneficial and detrimental effects post-TBI, and general immunosuppression has not proven helpful (Russo and McGavern, 2016; Loane and Byrnes 2010). Neuroinflammation has also been implicated in many neurodegenerative diseases such as Parkinson's and Alzheimer's disease (Faden and Loane, 2015). Fully understanding the mechanisms behind the body's neuroinflammatory response will not only allow for the development of targeted treatments for TBI, but also will likely allow for better treatments for those with neurodegenerative diseases (Ransohoff, 2016) and other diseases that may have a neuroinflammatory component such as diabetes (Wang et al 2014).

The epigenetic mechanisms that control gene expression of neuroinflammation have been studied, specifically the mechanism of histone deacetylases (HDACs). HDACs are a class of enzymes that modify chromatin conformation by removing acetyl groups from the histones of chromatin. This condenses chromatin, making it more difficult for the DNA to be accessed by transcriptional machinery and therefore repressing transcription (Allfrey et al 1964). In recent years, there has been a focus on HDACs as a therapeutic target to treat a variety of diseases including cancer, cardiovascular disease, stroke, injury and diabetes (Yoon and Eom, 2016; Wang et al, 2014; Mihylova et al, 2011 ; Baltan et al, 2011). Of the HDAC enzymes, class Ila HDACs receive particular attention given that they have higher expression in the brain and are involved in neuronal activity, degeneration, and apoptosis. Class Ila HDACs have been implicated as important factors in regulating the immune response (Altman and Kong, 2011 ; Poralla et al, 2015; Shakespear et al, 2013; Wang et al, 2014; Gupta et al, 2016). For example, HDAC5 and HDAC7 have been detected in macrophages, immortalized microglia known as BV2 cells, and microglia (Gupta et al 2016; Shakespear et al 2013; Crockett et al 2017). HDAC7 serves as a negative regulator of the anti-inflammatory gene, Nur77 (Dequiedt et al 2003; Hanna et al 2011; Liu et al 2017), and there is preliminary evidence of increased expression of HDAC7 in microglia following TBI (Crockett et al 201 ).

It has been hypothesized that class Ila HDACs may play an important role in initiating the neuroinflammatory response. Because of this, it is important to determine possible upstream factors that regulate class Ila HDAC function. Following this, it is important to examine the possibility that polarity proteins Parl (partitioning defective 1), also known as MARK 
(microtubule affinity regulating kinase), act as functional regulators of HDAC Ila. This is because MARK2/Par-1b has been observed to play a vital role in neuronal migration and axon formation. It was previously observed that animals deficient in Par-1b/MARK2 seemed to be "primed" for an inflammatory response; Par-1b/MARK2 deficient mice that had sustained TBI displayed a heightened inflammatory response (DiBona et al 2019). It was therefore proposed that one of MARK2's downstream functions is to regulate class Ila HDACs within microglia and their response to injury. By determining whether such a signaling pathway exists within microglia, it is possible that this pathway could be therapeutically targeted in the future with the hope of regulating the runaway inflammatory response associated with brain injury.

\section{Materials and Methods}

To ensure the rigor and reproducibility of the studies, the following measures were taken. First, data collection and analyses were completely blinded to the experimenter. Second, rigorous control experiments were performed to ensure the validity of the conclusions-all antibodies were positive and negative control tested with immunohistochemistry.

Our TBI studies were conducted using a controlled cortical impact $(\mathrm{CCl})$ injury model in mice over the course of several years (e.g., DiBona et al 2019). Sham mice (those who just had a craniotomy performed without significant injury) as well as $\mathrm{CCl}$-injured mice consisting of both wild-type (WT) and Par1 b/MARK2 knockout (KO) (-/-) mice (B6.129X1-Mark2tm1 Hpw/J; stock 009365) were used. Knockout mice (without Par1b/MARK2) were used to demonstrate an in vivo model of changes in morphology and density of astrocytes and microglia. All animal procedures were approved by the Rutgers-RWJMS Institutional Animal Care and Use Committee prior to the start of experimentation. All animals had free access to food and water and were housed in a clean, temperature-regulated facility that maintained a strict 12-hour light/dark cycle. Animals were anesthetized prior to being sacrificed by cardiac perfusion. Once the mice were phosphate-buffered saline (PBS) cleared and fixed with paraformaldehyde (PFA), the brains were extracted and submerged in PFA for 1-2 hours post-fixing. Each brain was washed with PBS and transferred through sucrose gradients (10\%, 20\%, and 30\%) for one day each and stored in a $4^{\circ} \mathrm{C}$ refrigerator.

Brains from these mice were mounted with O.C.T. Compound embedding medium, and $60-\mu \mathrm{m}$ sections were obtained with a frozen sliding microtome. Brains were pierced on the right side (injury was made on the left) to serve as a landmark and maintain orientation throughout the later steps of the process. Sections were stored in cryoprotectant solutions (25\% Glycerol, 25\% Ethylene Glycol, 30\% Sucrose in $0.1 \mathrm{M}$ PBS) until immunohistochemistry was performed. For immunohistochemistry, two intact hippocampal sections per animal were taken. Cells were stained with DAPI, GFAP, and IBA1. DAPI is a stain for gross cell morphology and is used to label nuclei. GFAP is a stain used to determine astrocyte activation, as astrocytes are specialized cells 
that function to support the central nervous system (CNS). IBA1 is a stain used to determine microglia activation, as microglia are specialized macrophages of the CNS.

For the $\mathrm{CCl}-\mathrm{TBI}$ experiment, tissue was washed 3 times for 5 minutes each with $1 \times$ PBS to remove cryoprotectant residue. Following thorough washing, tissue was permeabilized in Blocking Solution ( $2 \mathrm{~mL}$ Donkey Serum, $50 \mathrm{~mL}$ PBS-T) for 30 minutes at room temperature. After blocking Solution was removed from each tissue, the unrinsed sham-operated control and $\mathrm{CCl}-\mathrm{TBI}$ tissues were then incubated with $0.200 \mathrm{~mL}$ of donkey-anti-mouse Fab fragment IgC (Jackson ImmunoResearch, West Grove, PA, 1:60 dilution) for at least 1 hour at room temperature. This solution was removed from each tissue, and primary antibodies were then applied at the desired dilution to incubate overnight at room temperature. The following day, tissue was washed again using the process above, secondary antibody at desired dilution was applied, and the tissues were incubated at room temperature for at least 2 hours. The tissue was then washed and mounted on slides, before being sealed in with DPX. To minimize bias in imaging acquisition and quantification, all slides were blinded prior to imaging, and unblinding occurred after image quantification.

\section{Results}

These figures are fluorescent microscope images taken after immunohistochemistry was performed on brain sections (containing the hippocampus) taken from different mice. Figure 1 acts as a summary of findings in the ipsilesional cortex taken from brain of a mouse with closed head injury $(\mathrm{CHI})$ performed at 21 days and euthanization performed at 28 days. In Figure $1 \mathrm{~A}$, the DAPI stain demonstrates that there are no obvious changes to the structure of the cortex or hippocampus other than the injury site, which negates the possibility of increased expression being due to structural changes. However, in Figure $1 \mathrm{~B}$ and $\mathrm{C}$, the results qualitatively indicate an increase in expression of GFAP (Figure 1 B) and IBA1 (Figure $1 \mathrm{C}$ ). This is made more evident when looking at Figure 2, which serves as a negative control and shows the baseline levels of expression for both GFAP and IBA1 in CHI092319-1, a MARK2 wild-type sham mouse (only craniotomy) operated on at 20 days and euthanized at 28 days. 

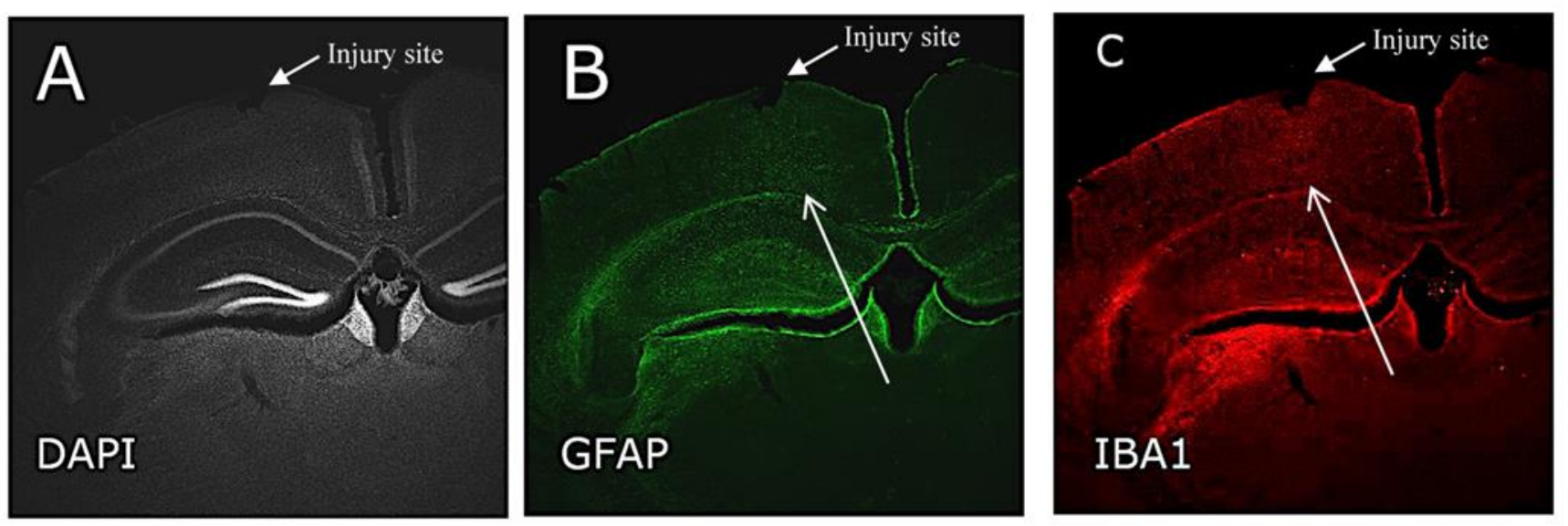

Figure 1: Fluorescent microscopy imaging of ipsilesional part of hippocampal section taken from brain of a mouse with closed head injury (CHI) performed at 21 days, and euthanized at 28 days. A DAPI staining serves as control, showing cortical brain structure and injury site. B Arrow indicates increased expression of GFAP in cortex. C Arrow indicates increased expression of IBAl in cortex.

Figure 2
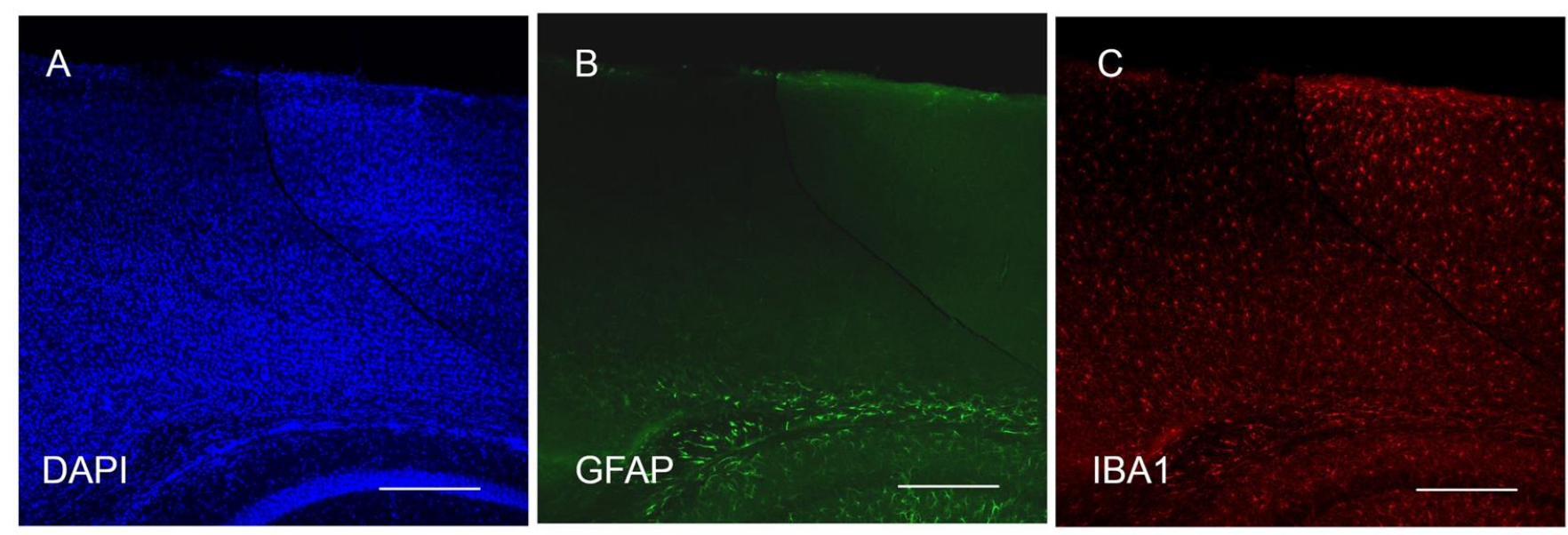

Figure 2: Fluorescent microscopy imaging of hippocampal section of brain taken from a sham (no CHI) performed at 20 days, and euthanized at 28 days. A DAPI staining showing no injury site. B Baseline levels of GFAP expression. C Baseline levels of IBA 1 expression.

Figure 3 shows the differences in expression levels on each side of the cortex: the same side as the injury (ipsilesional) and the opposite side of the injury (contralesional). There is increased expression of GFAP in both the ipsilesional (Figure $1 \mathrm{~B}$ ) and contralesional (Figure $1 \mathrm{C}$ ) sides of the cortex; however, the ipsilesional side has a much greater increase in expression in GFAP, especially in the area immediately surrounding the injury site. There is also increased expression of IBA1 in both the ipsilesional (Figure $1 \mathrm{E}$ ) and contralesional (Figure $1 \mathrm{~F}$ ) sides of the cortex; however, the ipsilesional side has a much greater increase in expression in IBA1, especially in the area immediately surrounding the injury site. 


\section{Discussion}

\section{Purpose}

The purpose of this project was to determine the role that class Ila HDACs may play in initiating the inflammatory response after TBI. Now that it is understood that class Ila HDACs, particularly HDAC7, do in fact play a role in initiation of the inflammatory response after TBI, upstream factors can be explored to unearth the molecular mechanism behind the subsequent neuroinflammation after TBI. Gaining knowledge of the pathways involved will facilitate attempts to discover therapy or even cures for TBI and spinal cord injury (SCl).

Though it took several tries to obtain intact sections and perform operational IHC staining, the experiment was successful since the high expression of GFAP and IBAI supported the hypothesis that HDAC plays a role in mediating neuroinflammation. This is evident through the IHC staining, which involved mixing HDAC7 (a class Ila HDAC) with GFAP and IBAI secondary antibodies. When comparing injury in Figure 1 vs sham in Figure 2 results, there is a higher expression of GFAP and IBAI in the closed head injury mouse compared to the baseline levels in the sham operation mouse. The results also suggest something that had no clearly defined expectations, which was a global increase in expression of GFAP and IBA1. This is evident in Figure 3, when comparing the contralesional and ipsilesional sides of the cortex. Not only was there increased expression of GFAP and IBAI in the same side of the cortex as the injury, but also on the opposite side of the brain (albeit in lower concentration). This indicates that the inflammation doesn't just take place at the injury site, but spreads throughout the rest of the brain at the molecular level. The reason this increased staining is important is because of the roles these cells play in the CNS. If there is disease or injury (such as $\mathrm{SCl}$ or TBI) of the CNS, one would expect an activation in astrocytes (given their role of support) and microglia (given their role of maintaining CNS health by phagocytizing infection and damaged neurons). 
Figure 3

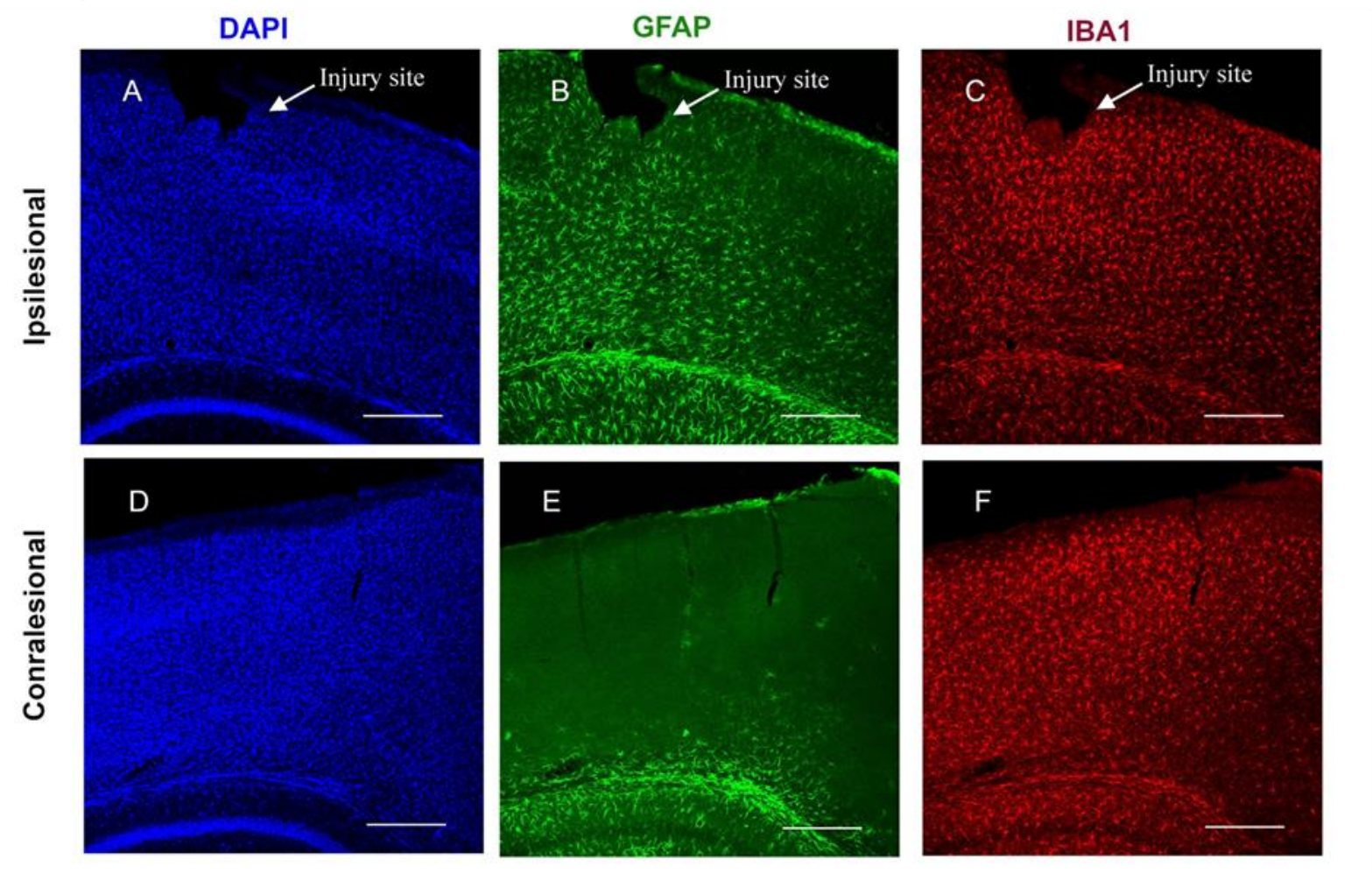

Figure 3: Fluorescent microscopy imaging of ipsilesional and contralesional cortex in a hippocampal section of brain taken from MARK WT mouse CHIO92419-1, with closed head injury (CHI) performed at 21 days and euthanization performed at 28 days. Scale Bars $=250$ um. A DAPI staining serves as control, showing ipsilesional cortical brain structure. B Arrow indicates increased expression of GFAP in the ipsilesional cortex. $C$ Arrow indicates increased expression of IBAI in the ipsilesional cortex. DDAPI staining serves as control, showing contralesional cortical brain structure. E Arrow indicates increased expression of GFAP in the contralesional cortex. FArrow indicates increased expression of IBAI in the contralesional cortex

\section{Future directions}

The next stage of this experiment is to compare the expression level differences between the contralesional and ipsilesional sides of the cortex in a MARK2-deficient mouse with $\mathrm{CHI}$, as well as expression levels in a CHI MARK2-deficient mouse vs sham MARK2-deficient mouse. When these molecular results are obtained and compared to the results from these wildtype (WT) mice, it can be determined whether Par1 b/MARK2 is a possible upstream factor for class Ila HDACs.

After this is completed, the next steps will be to test possible therapies from both behavioral and molecular perspectives. The potential therapies for TBI that will be focused on are the diabetic drug Metformin and class Ila HDAC inhibitors. The diabetic drug Metformin has been 
identified as a potential candidate because of its anti-oxidative, anti-inflammatory, and antiischemic properties, which may lend itself to neuroprotective effects. There is evidence that Metformin can improve the cognitive outcome of mice following TBI (DiBona et al, under review). These studies suggested that Metformin's pleiotropic effects (Bridgeman et al, 2018) may affect the neuroinflammatory response through stimulation of Par $1 \mathrm{~b}$. Even a $50 \%$ decrease in Par1b/Mark2 can lead to an apparent increase in neuroinflammation (DiBona et al 2019). Metformin treatment has shown positive effects in several animal studies dealing with spinal cord injury and brain trauma (Wang et al 2016; Zhang et a 2016; Tao et al 2018; DiBona et al 2019 under review) and in human clinical trials (Taheri et al 2019). In addition, Metformin has been shown to influence HDAC7 functioning (Mihaylova et al 2011; Bridgeman et al; 2018). Metformin has been shown to alter microglial functioning in an animal model of stroke (Ji et al, 2018). Although the mechanism by which Metformin creates positive effects in injury models is not fully understood, it has been hypothesized that Metformin dampens the inflammatory response by decreasing the availability of nuclear HDAC7 by enhancing Par1b/MARK2 activity in microglia.

\section{Possible flaws}

One of the potential problems with this protocol is the sham operation animal as a negative control. According to our experimental setup, the sham mouse receives a craniotomy in place of a closed head injury. Though the craniotomy did function as a negative control in this experiment, we cannot be sure that a craniotomy procedure does not cause unique trauma different from that of $\mathrm{CHI}$ and accompanying unique neuroinflammation. As a result, this may not be the best representation of baseline expression levels of GFAP and IBA1. To prevent such confounding data disrupting the results of other experiments, a future experiment could be done comparing an animal only under anesthesia with the conventional negative control of sham operation. This experiment would demonstrate whether the sham is an accurate representation of baseline expression levels without conferring distinct damage and, therefore, altered expression levels.

\section{Acknowledgements}

Special thanks to Dr. David Crockett for serving as a wonderful mentor and providing many great learning opportunities. In addition, many thanks to the Aresty Reseach Center for funding this research project and to Robert Wood Johnson Medical School for providing resources. This paper was a progress report submitted to The Department of Cell Biology and Neuroscience Rutgers University. 


\section{References}

Allfrey, V. G., Faulkner, R., \& Mirsky, A. E. (1964) Acetylation \& methylation of histones \& their possible role in the regulation of RNA synthesis. Proceedings of the National Academy of Sciences. (5): $51786--794$.

Altman, Amnon \& Kong, Kok-Fai (2011) To kill, you have to duck an HDAC. Nature Immunology. :12279 EP -.

Baltan, Selva, Bachleda, Amelia, Morrison, Richard S, \& Murphy, Sean P (2011) Expression of histone deacetylases in cellular compartments of the mouse brain $\&$ the effects of ischemia. Trans/ Stroke Res. (3):2411-23.

Bridgeman, Stephanie Claire, Ellison, Gaewyn Colleen, Melton, Phillip Edward, Newsholme, Philip, \& Mamotte, Cyril Desire Sylvain (2018) Epigenetic effects of metformin: From molecular mechanisms to clinical implications. Diabetes, Obesity \& Metabolism.

(7):201553--1562.

Das Gupta, Kaustav, Shakespear, Melanie R, Iyer, Abishek, Fairlie, David P, \& Sweet, Matthew J (2016) Histone deacetylases in monocyte/macrophage development, activation \& metabolism: refining HDAC targets for inflammatory \& infectious diseases. Clinical \& Translational Immunology. (1):5e62.

Dequiedt, F. , Martin, M. , Von Blume, J. , Vertommen, D. , Lecomte, E. , Mari, N. , Heinen, M.-F. , Bachmann, M. , Twizere, J.-C. , Huang, M. C. , \& et al. (2006)New Role for hPar-1 Kinases EMK \& C-TAK1 in Regulating Localization \& Activity of Class Ila Histone Deacetylases. Molecular \& Cellular Biology. (19):267086--7102.

Dequiedt, Franck, Kasler, Herbert, Fischle, Wolfgang , Kiermer, Veronique , Weinstein, Marc , Herndier, Brian G , \& Verdin, Eric (2003)HDAC7, a thymus-specific class II histone deacetylase, regulates Nur77 transcription \& TCR-mediated apoptosis. Immunity. (5): 18687-98.

Dewan, Michael C , Rattani, Abbas , Gupta, Saksham , Baticulon, Ronnie E, Hung, Ya-Ching , Punchak, Maria , Agrawal, Amit , Adeleye, Amos O , Shrime, Mark G , Rubiano, Andrés M , Rosenfeld, Jeffrey $V$, \& Park, Kee B (2018)Estimating the global incidence of traumatic brain injury. J Neurosurg. :1-18.

DiBona, Victoria L , Zhu, Wenxin , Shah, Mihir K, Rafalia, Aditi , Ben Cheikh, Hajer , Crockett, David P , \& Zhang, Huaye (2019)Loss of Par1b/MARK2 primes microglia during brain development \& enhances their sensitivity to injury. J Neuroinflammation. (1):1611.

Faden, Alan I. \& Loane, David J. (2014) Chronic Neurodegeneration After Traumatic Brain Injury: Alzheimer Disease, Chronic Traumatic Encephalopathy, or Persistent Neuroinflammation? Neurotherapeutics. (1):12143--150.

Hanna, Richard N. , Shaked, Iftach , Hubbeling, Harper G. , Punt, Jennifer A. , Wu, Runpei , Herrley, Erica, Zaugg, Claudia, Pei, Hong, Geissmann, Frederic, Ley, Klaus , \& et al. (2012)NR4A1 (Nur77) Deletion Polarizes Macrophages Toward an Inflammatory Phenotype \& Increases Atherosclerosis. Circulation Research. (3):1 10416--427. 
Ji, Juan, Xue, Teng-Fei , Guo, Xu-Dong , Yang, Jin , Guo, Ruo-Bing , Wang, Juan, Huang, Ji-Ye , Zhao, Xiao-Jie , \& Sun, Xiu-Lan (2018)Antagonizing peroxisome proliferator-activated receptor $\gamma$ facilitates $M 1$-to-M2 shift of microglia by enhancing autophagy via the LKB1AMPK signaling pathway. Aging Cell. (4):17e12774.

Liu, Tian-Ya, Yang, Xiao-Ying , Zheng, Long-Tai, Wang, Guang-Hui , \& Zhen, Xue-Chu (2016)Activation of Nur77 in microglia attenuates proinflammatory mediators production $\&$ protects dopaminergic neurons from inflammation-induced cell death. Journal of Neurochemistry. (4):140589--604.

Loane, David J \& Byrnes, Kimberly R (2010) Role of microglia in neurotrauma. Neurotherapeutics. (4):7366-77.

Mihaylova, Maria M. , Vasquez, Debbie S. , Ravnskjaer, Kim , Denechaud, Pierre-Damien, Yu, Ruth T. , Alvarez, Jacqueline G. , Downes, Michael , Evans, Ronald M. , Montminy, Marc , \& Shaw, Reuben J. (2011)Class Ila Histone Deacetylases Are Hormone-Activated Regulators of FOXO \& Mammalian Glucose Homeostasis. Cell. (4):145607--621.

Poralla, Lukas, Stroh, Thorsten, Erben, Ulrike, Sittig, Marie, Liebig, Sven, Siegmund, Britta, \& Glauben, Rainer (2015) Histone deacetylase 5 regulates the inflammatory response of macrophages. Journal of Cellular \& Molecular Medicine. (9):192162--2171.

Ransohoff, Richard M. (2016) How neuroinflammation contributes to neurodegeneration. Science. (6301):353777--783.

Russo, Matthew V. \& McGavern, Dorian B. (2016) Inflammatory neuroprotection following traumatic brain injury. Science. (6301):353783--785.

Shakespear, Melanie R. , Hohenhaus, Daniel M. , Kelly, Greg M. , Kamal, Nabilah A. , Gupta, Praveer, Labzin, Larisa I. , Schroder, Kate , Garceau, Valerie , Barbero, Sheila , Iyer, Abishek, \& et al. (2013)Histone Deacetylase 7 Promotes Toll-like Receptor 4-dependent Proinflammatory Gene Expression in Macrophages. Journal of Biological Chemistry. (35):28825362--25374.

Taheri, Ali , Emami, Mahdi , Asadipour, Erfan , Kasirzadeh, Sara , Rouini, Mohammad-Reza , Najafi, Atabak, Heshmat, Ramin, Abdollahi, Mohammad , \& Mojtahedzadeh, Mojtaba (2019)A randomized controlled trial on the efficacy, safety, \& pharmacokinetics of metformin in severe traumatic brain injury. Journal of Neurology. (8):2661988--1997.

Wang, Chen , Liu, Chang, Gao, Kai , Zhao, Haosen, Zhou, Zipeng , Shen, Zhaoliang , Guo, Yue , Li, Zhuo , Yao, Tianchen , \& Mei, Xifan \& (2016)Metformin preconditioning provide neuroprotection through enhancement of autophagy $\&$ suppression of inflammation $\&$ apoptosis after spinal cord injury. Biochemical \& Biophysical Research Communications. (4):477534--540.

Xu, Ge, Ge, Yinggang, Tao, Xiaohong, Gao, Qing, \& Liang, Xiaoyan (2017) MARK2 inhibits the growth of HeLa cells through AMPK \& reverses epithelial-mesenchymal transition. Oncology Reports. (1):38237--244.

Yoon, Somy \& Eom, Gwang Hyeon (2016) HDAC \& HDAC Inhibitor: From Cancer to Cardiovascular Diseases. Chonnam medical journal. (1):521--11.

Zhang, Di , Xuan, Jun, Zheng, Bin-bin, Zhou, Yu-long, Lin, Yan , Wu, Yao-sen, Zhou, Yi-fei , Huang, Yi-xing, Wang, Quan, Shen, Li-yan , \& et al. (2016) Metformin Improves 
Aresty Rutgers Undergraduate Research Journal, vol. 1, issue 1, Spring 2020

Functional Recovery After Spinal Cord Injury via Autophagy Flux Stimulation. Molecular Neurobiology. (5):543327--3341. 